\title{
Association Between Culture-Negative Versus Culture-Positive Sepsis and Outcomes of Patients Admitted to the Pediatric Intensive Care Unit
}

Tarek R. Hazwani ${ }^{1,2}$, Yasser M. Kazzaz ${ }^{3}$, Shaden Alsugheir ${ }^{4}$, Shahad Aldelaijan ${ }^{5}$, Futoon Alsugheir ${ }^{4}$, Hamza Alali ${ }^{3}$, Alaa Alsadoon ${ }^{6}$, Moudi Alhamwah ${ }^{4}$, Sara Alsubaiel ${ }^{4}$, Bassam Alomar ${ }^{7}$, Ramesh Vishwakarma ${ }^{8}$

1. Pediatric Intensive Care, Ministry of National Guard - Health Affairs, Riyadh, SAU 2. College of Medicine, King Saud Bin Abdulaziz University for Health Sciences, Riyadh, SAU 3. Pediatric Critical Care, Ministry of National Guard Health Affairs, Riyadh, SAU 4. Pediatrics, Ministry of National Guard - Health Affairs, Riyadh, SAU 5. Emergency Medicine, Ministry of National Guard - Health Affairs, Riyadh, SAU 6. Pediatric Infectious Diseases, Ministry of National Guard - Health Affairs, Riyadh, SAU 7. Pediatric Emergency Medicine, Ministry of National Guard - Health Affairs, Riyadh, SAU 8. Biostatistics, King Abdullah International Medical Research Center, Riyadh, SAU

Corresponding author: Tarek R. Hazwani, tarekhazwani@gmail.com

\section{Abstract \\ Background}

Sepsis remains a major cause of death, with high mortality and morbidity rates in children. The cause of mortality may be associated with several factors, including differences in cultures and the type of organism. This study was aimed at evaluating the characteristics and outcomes of negative bacterial blood culture compared to those of positive bacterial blood culture in children with severe sepsis/septic shock.

\section{Methods}

A retrospective cohort study was conducted at a pediatric intensive care unit (PICU) of a tertiary care medical center. All pediatric patients, from newborn to 14 years of age, admitted between April 2015 and January 2018 were included in the study if they fulfilled the criteria for severe sepsis/septic shock.

\section{Results}

Of the 209 patients, 30 (14.3\%) had a positive bacterial blood culture whereas 179 (86.6\%) had a negative bacterial blood culture. Mortality was more in positive bacterial blood culture $13(43 \%)$ vs 35 (20\%) in negative bacterial blood culture $(\mathrm{P}=0.004)$. Respiratory tract infections were extremely common, present in 108 of $179(60 \%)$ patients, and tended to result in a negative culture. The rate of organ dysfunction was higher in the positive bacterial blood culture group at admission $(\mathrm{P}=0.01)$. However, the results did not reveal a significant finding related to multiorgan dysfunction syndrome (MODS) progression over three days of PICU admission $(\mathrm{P}=0.06)$.

Received 08/11/2020

Review began 08/13/2020 Review ended 08/23/2020 Published 08/24/2020

(c) Copyright 2020

Hazwani et al. This is an open access article distributed under the terms of the Creative Commons Attribution License CC-BY 4.0., which permits unrestricted use, distribution, and reproduction in any medium, provided the original author and source are credited.

\section{Conclusion}

The negative bacterial blood culture constitutes a substantial proportion of pediatric patients with severe sepsis/septic shock. Furthermore, these pediatric patients have a lower mortality rate compared to positive bacterial blood cultures. The culture-negative sepsis group also had less organ dysfunction.

Categories: Pediatrics, Infectious Disease

Keywords: sepsis, culture, outcome, organ dysfunction, pediatric

\section{Introduction}

Severe sepsis/septic shock is a life-threatening condition, commonly managed at pediatric intensive care units (PICUs) worldwide. It is a major cause of morbidity and mortality in children, with a mortality rate of over $25 \%$ among patients in hospital admission with severe sepsis. It is estimated to affect 22 children less than 18 years of age per 100,000 person-years [1,2]. This exerts a significant impact on PICU utilization as $8 \%$ of children in PICUs meet the criteria for severe sepsis [1,3]. Most cases of mortality occur in the first 4872 hours of PICU admission, mainly due to refractory shock and/or multiorgan dysfunction syndrome (MODS) [4,5]. Therefore, the early recognition and prediction of who are at high risk is a major contributor to sepsis morbidity and mortality and allow for timely changes in the management at PICUs, improving the overall outcomes [2].

Sepsis in children represents a spectrum of diseases involving the systemic inflammatory response syndrome in the setting of infections, escalating in septic shock to cardiovascular and organ system dysfunction in critically ill patients. Pediatric logistic organ dysfunction is a pediatric organ dysfunction 
score system validated for the severity classification of patients with sepsis $[6,7]$. The clinical course of septic shock is affected by the age, immune state of the patient, virulence of the pathogen, and hemodynamic adaptation to circulatory failure.

Although the definition and management of sepsis have changed dramatically over the last few years, the prediction of mortality of sepsis in children remains a challenge. Many markers have been studied and evaluated, such as lactate, chronic disease, cardiovascular, and respiratory derangements at PICU admission [4,8-11]. However, mortality in pediatric sepsis is mostly attributable to MODS, respiratory failure, or neurologic injury [5].

Blood culture is fundamental to the definition of sepsis and further management in children with suspected sepsis. However, blood cultures in patients with suspected sepsis are often negative, and isolation of specific organisms by culture remains challenging. Although $28 \%-49 \%$ of cases of severe sepsis in adults have been described to be culture-negative, data are limited on the epidemiology and outcomes in the pediatric population of those with culture-negative severe sepsis (CNSS) [12].

A limitation of larger studies on pediatric sepsis is the use of clinical definitions of sepsis, with only a few sepsis episodes being confirmed microbiologically and scant robust characterization of the presence and effect of organ dysfunction on outcomes $[3,13]$.

Despite the publication of only a few studies that investigated the association of a positive blood culture with mortality in the pediatric population, the predictive significance of a positive blood culture in children with sepsis admitted to the PICU remains uncertain [14].

The aim of our study was to describe the patterns of culture-positive bacteria in relation to the incidence, pathogens, severity, organ dysfunctions, and outcomes to determine if a positive bacterial blood culture predicted increased morbidity and mortality among patients admitted to the PICU with severe sepsis.

Our goals were:

1- Investigate the proportion and trends of CNSS among severe sepsis patients admitted to the PICU.

2- Investigate whether or not being culture-positive is an independent predictor of mortality in patients with severe sepsis.

\section{Materials And Methods \\ Design, setting, participants, and variables}

We conducted a retrospective cohort study at King Abdullah Specialized Children's Hospital, a tertiary care medical center under the Ministry of National Guard - Health Affairs in Saudi Arabia, with 25 beds at the PICU involving medical and surgical cases. The present research comprises a pre-specified ancillary study of the original study: association of antibiotics administration timing with mortality in children with sepsis in a tertiary care hospital of a developing country.

Using the aforementioned database, patients aged 0 to 14 years who had been admitted between April 2015 and January 2018 were included in the study if they fulfilled the criteria for severe sepsis/septic shock. Children admitted to a separate neonatal or cardiac intensive care unit and those with advanced directives limiting resuscitation therapies at the time of sepsis recognition were excluded.

We extracted data derived from electronic medical records of pediatric sepsis patients that were collected and manually reviewed. The following variables were collected: patient demographics, Pediatric Index of Mortality 3 (PIM 3) score [15], comorbidities, hospital length of stay (LOS), PICU LOS, clinical and management characteristics (vasoactive agents and mechanical ventilation), MODS, source of infection, and culture results (blood, cerebrospinal fluid, urine, and respiratory examinations).

\section{Data sources and definitions}

We enrolled patients with severe sepsis/septic shock, as defined by the International Pediatric Sepsis Consensus Conference (IPSCC), or diagnosed by the treating physician [16]. Patients were identified using the PICU administrative database and medical records with International Classification of Diseases sepsisrelated codes [17]. Organ dysfunction was defined as the IPSCC criterion [16].

CNSS was determined based on the diagnosis of severe sepsis/septic shock in the absence of positive bacterial pathogen cultures from blood. We categorized comorbidities according to the pediatric complex chronic conditions classification system, version 2 [18].

\section{Ethical statement}


This study was approved by the Institutional Review Board (IRB) of the King Abdullah International Medical Research Center (RC19/235/R). A waived consent protocol was approved by the IRB.

\section{Interventions}

Our study focused on the characteristics and outcomes of patients with CNSS compared to those with culture-positive severe sepsis (CPSS). The primary outcome measures were the proportion of patients with severe sepsis who had a negative culture, trend of CNSS patients admitted to the PICU, and incidence and trends of mortality associated with CPSS in comparison with CNSS. As for secondary outcomes, we investigated if being culture-positive is associated with more number of ventilation days and MODS incidence compared to CNSS.

\section{Statistical analysis}

All data were analyzed using SAS, version 9.3 (SAS Institute, Cary, NC), coded and de-identified, and cleaned from missing data and duplicates. Patient demographics, clinical characteristics, and outcomes are expressed as median and percentile (Q1, Q3) to describe the continuous variables of age, PIM 3 score, and progression of organ dysfunction. Frequencies and percentages were used to describe the categorical variables, such as sex, source of infection, organ dysfunction, number of comorbidities, and management (mechanical ventilation and vasopressors). PICU and hospital LOS were censored after 30 days for children still in the PICU or hospital on day 30 and for patients who died. The ventilation-free days were defined as the number of days between successful extubation and day 28 ; therefore, it was considered zero if the patient died before 28 days.

The chi-square test was used for correlation, and logistic regression was used to predict mortality among those who had positive and negative blood cultures. A P-value $<0.05$ was considered statistically significant.

\section{Results}

\section{Demographics and trends of CNSS}

During the study period, 206 children were admitted to the PICU with severe sepsis/septic shock. There was an almost equal sex distribution. The patients' median (Q1, Q3) ages were $19.5(3,73)$ and $11.5(3,63)$ months in the positive and negative bacterial blood culture groups, respectively. There were no statistically significant differences in the age, sex, PIM score, or source of admission between the two groups. Of the total patients, $30(14.3 \%)$ had a positive bacterial blood culture whereas $179(86.6 \%)$ had a negative bacterial blood culture (Table 1). 


\section{Cureus}

\begin{tabular}{|c|c|c|c|c|}
\hline \multicolumn{2}{|l|}{ Characteristic } & Positive Bacterial Blood Culture ( $\mathrm{N}=30$ ) & Negative Bacterial Blood Culture ( $N=179)$ & P-value \\
\hline \multicolumn{2}{|l|}{ Age (months) } & $11.5(3,63)$ & $19.5(3,73)$ & $0.52^{\wedge}$ \\
\hline \multicolumn{2}{|c|}{ Neonate (less than one month) } & $4(13.3)$ & $24(13.4)$ & \multirow{3}{*}{$0.3^{\wedge \pi}$} \\
\hline \multicolumn{2}{|c|}{ Infant (1 month - Below one year) } & $11(36.7)$ & $46(25.7)$ & \\
\hline Children (1-14 years) & & $15(50)$ & $109(60.9)$ & \\
\hline \multirow{2}{*}{ Gender } & Male & $12(40)$ & $88(49.2)$ & \multirow{2}{*}{$0.35^{\wedge \wedge}$} \\
\hline & Female & $18(60)$ & $91(50.8)$ & \\
\hline \multicolumn{2}{|l|}{ PIM 3 score } & $0.0(0.02,0.09)$ & $0.0(0.01,0.11)$ & $0.71^{\wedge}$ \\
\hline \multirow{2}{*}{ Source of admission } & ER & $22(73.3)$ & $129(72.1)$ & \multirow{2}{*}{$0.89^{\wedge \wedge}$} \\
\hline & Ward & $8(26.7)$ & $50(27.9)$ & \\
\hline \multirow{4}{*}{ Comorbidities } & 0 & $5(16.7)$ & $36(20.1)$ & \multirow{4}{*}{$0.24^{\wedge \wedge}$} \\
\hline & 1 & $17(56.7)$ & $69(38.5)$ & \\
\hline & 2 & $5(16.7)$ & $34(19.0)$ & \\
\hline & $>3$ & $3(10)$ & $40(22.3)$ & \\
\hline
\end{tabular}

\section{TABLE 1: Demographics}

$\wedge^{\wedge}$ Chi-square test is used to calculate the P-value.

${ }^{\wedge}$ Mann Whitney-U test is used to calculate the P-value.

PIM 3: Pediatric Index of Mortality 3

\section{Patient characteristics based on the blood culture status}

With respect to comorbidities, there were no statistically significant differences between the two groups. The distribution of new-onset acute organ dysfunction varied between the two groups. Significantly fewer patients with a negative bacterial blood culture had organ dysfunction at the time of PICU admission (median, 2.9 vs. $3.0, \mathrm{P}=0.02$ ). All organ dysfunctions, namely cardiac, respiratory, neurological, hematological, renal, and hepatic dysfunctions, were higher in the CPSS group. However, the results did not reveal a significant finding related to MODS progression over three days of PICU admission (median, 0.0 vs. 1.0, $\mathrm{P}=0.06$ ) (Tables 2, 3).

\begin{tabular}{|c|c|c|c|}
\hline Characteristic & Positive Bacterial Blood Culture $(\mathrm{N}=30)$ & Negative Bacterial Blood Culture $(\mathrm{N}=179)$ & P-value \\
\hline Organ dysfunction on time zero & $3.0(2,5)$ & $2.9(2,4)$ & 0.02 \\
\hline Organ dysfunction during 3 days & $0.0(-1,1)$ & $-1.0(-2,0.0)$ & 0.06 \\
\hline
\end{tabular}

\section{TABLE 2: Multiple organ dysfunction progression}

Mann Whitney-U test is used to calculate the P-value. 


\section{Cureus}

\begin{tabular}{|c|c|c|c|}
\hline & Positive Bacterial Blood Culture & Negative Bacterial Blood Culture & P-Value \\
\hline Mortality & $13(43.3)$ & 35 (19.6) & $0.004^{\wedge} \wedge$ \\
\hline Duration of mechanical ventilation, days & $5.0(1,10)$ & $7.0(4,12)$ & $0.04^{\wedge}$ \\
\hline Duration of PICU stay, days & $7.0(1,11)$ & $6.0(2,13)$ & $0.95^{\wedge}$ \\
\hline Duration of hospital stay, days & $18.5(8,61)$ & $15.0(8,37)$ & $0.66^{\wedge}$ \\
\hline Delta MODS Day 0 to 3 & $0.0(-1,1)$ & $-1.0(-2,0.00)$ & $0.06^{\wedge}$ \\
\hline Ventilation free days & $0.0(0.00,22)$ & $18.0(0.00,23)$ & $0.09^{\wedge}$ \\
\hline Inotropes Days & $2.5(1,5)$ & $3.0(2,6)$ & $0.35^{\wedge}$ \\
\hline
\end{tabular}

\section{TABLE 3: Outcomes: positive bacterial blood culture vs. negative bacterial blood culture}

$\wedge \wedge$ Chi-square test is used to calculate the P-value.

${ }^{\wedge}$ Mann Whitney-U test is used to calculate the P-value.

The incidence of primary bloodstream infection, and no positive culture at another body site, was found to be significantly higher in the CPSS group, seen in 16 of a total of 30 cases $(\mathrm{P}<0.0001)$ and it was the most common site of infection in our study. Respiratory tract infections were extremely common, present in 108 of 179 cases $(60 \%)$, and tended to result in a negative culture.

There was a statistically significant difference in the infection site between the positive and negative bacterial blood culture groups (Table 4).

\begin{tabular}{|c|c|c|c|}
\hline Infection site & Positive Bacterial Blood Culture $(\mathrm{N}=30$ ) & Negative Bacterial Blood Culture $(\mathrm{N}=179)$ & P-value \\
\hline Primary bloodstream infection & $16(53.3)$ & $7(3.9)$ & \multirow{8}{*}{$<0.0001$} \\
\hline Respiratory & $8(26.7)$ & $108(60.3)$ & \\
\hline Skin/soft tissue & $3(10.0)$ & $5(2.8)$ & \\
\hline Abdominal & 1 (3.3) & $17(9.5)$ & \\
\hline Genitourinary & 1 (3.3) & $10(5.6)$ & \\
\hline Unknown & 1 (3.3) & $28(15.6)$ & \\
\hline CNS & 0 & $3(1.7)$ & \\
\hline Endocarditis & 0 & $1(0.6)$ & \\
\hline
\end{tabular}

\section{TABLE 4: Infection sites based on culture status}

Fisher Exact test is used to calculate the P-value.

CNS: central nervous system

Patients with a negative bacterial blood culture did not differ significantly from patients with CPSS in terms of inotropes, ventilation-free days, PICU LOS, or hospital LOS (Table 3).

\section{Mortality based on the blood culture status}

Mortality was more in CPSS $13(43 \%)$ vs. $35(20 \%)$ in CNSS $(P=0.004)$ in our study, which indicated a higher proportion of deaths in the CPSS group. The mortality status between the positive and negative bacterial blood culture groups was found to be statistically significantly different $(\mathrm{P}=0.004)$ (Table 3$)$.

\section{Discussion}


Our study showed that only $30 \%$ of the children with severe sepsis/septic shock had a positive bacterial blood culture, which is lower than that in Morin et al.'s study [6]. The reasons for these findings may be attributed to the aggressive empiric outpatient therapy of infections and early administration of antibiotic therapy before cultures are drawn in patients when severe sepsis is recognized in the emergency room or in pediatric wards. Other potential explanations include increasing infections with viruses and fungi, particularly in immunocompromised patients, which may cause severe sepsis, as we investigated only the blood culture for bacterial organisms in our study.

In our study, patients with a negative bacterial blood culture had a significantly lower incidence of organ dysfunction at PICU admission. However, the progression of organ dysfunctions was statistically significant after three days of PICU admission, particularly cardiovascular and central nervous system failures and coagulopathy. These findings are consistent with the findings of other studies either in the pediatric or adult population [13,19], such as Lin et al.'s study, which showed that most pediatric patients with MODS developed it on day 1 (85\% of all patients with MODS) and that patients with MODS on day 1 were more likely to have bacteremia [20].

To the best of our knowledge, different sites of infections result in different prognoses of sepsis progression. The incidence of primary bloodstream infection was significantly higher in the positive bacterial blood culture group, seen in 16 of a total of 30 cases. In contrast to Agyeman et al.'s study [13], our results showed that CNSS was more common than CPSS in the presence of respiratory tract infections. These findings can be attributed to most respiratory tract infections in our patients who are diagnosed with viral infections.

Inotropic support is commonly required in patients with severe sepsis/septic shock admitted to the PICU; however, limited data could be found on the association between the blood culture status and inotrope requirement in pediatric patients with severe sepsis/septic shock. Schlapbach et al. found the highest mortality in children presenting with hypotension requiring vasopressors and lactate $>2 \mathrm{mmol} / \mathrm{L}$ (32.0\%) [4]. However, no statistically significant difference was observed in the use of inotropes in our results between the CPSS and CNSS groups.

Our results demonstrated that the mortality rate in the positive bacterial blood culture group was higher than that in the negative bacterial blood culture group, contrary to the study by Gupta et al. [21]. Our analysis differs from Gupta et al.'s study in several aspects. Our study included only bacterial organisms in blood culture results, and we did not include the viral and fungal results. As expected, bacterial sepsis was more severe and led to more MODS, which may explain our results. Moreover, our population was limited to children aged up to 14 years. Further investigations are warranted.

This study had some limitations. First, the data collection was retrospective. Therefore, there is a risk of missing data, and information bias is not always able to establish causation. Second, we restricted the analyses to bacterial cultures only. Expansion of the analyses to include other types of organisms, i.e., viruses and fungi, could be useful for understanding the association between the culture status and outcome. Third, this was a single-center study; therefore, we have to consider the differences in national health care systems and population demographics for its generalizability. Finally, using mortality as an outcome does not capture the impact of culture results on patients surviving with major long-term morbidity.

\section{Conclusions}

Our findings indicate that a negative bacterial blood culture constitutes a substantial proportion of pediatric patients with severe sepsis/septic shock. Furthermore, these pediatric patients have a lower mortality rate compared to positive bacterial blood cultures. The culture-negative sepsis group also had less organ dysfunction. A better understanding of bacterial sepsis in pediatrics and the reasons for worse associated outcomes of CPSS should be the focus of future studies.

\section{Additional Information}

\section{Disclosures}

Human subjects: Consent was obtained by all participants in this study. King Abdullah International Medical Research Center issued approval RC19/235/R. This study was approved by the Institutional Review Board (IRB) of the King Abdullah International Medical Research Center (RC19/235/R). A waived consent protocol was approved by the IRB. Animal subjects: All authors have confirmed that this study did not involve animal subjects or tissue. Conflicts of interest: In compliance with the ICMJE uniform disclosure form, all authors declare the following: Payment/services info: All authors have declared that no financial support was received from any organization for the submitted work. Financial relationships: All authors have declared that they have no financial relationships at present or within the previous three years with any organizations that might have an interest in the submitted work. Other relationships: All authors have declared that there are no other relationships or activities that could appear to have influenced the submitted work.

\section{References}


1. Weiss SL, Fitzgerald JC, Pappachan J, et al.: Global epidemiology of pediatric severe sepsis: the sepsis prevalence, outcomes, and therapies study. Am J Respir Crit Care Med. 2015, 191:1147-1157. 10.1164/rccm.201412-23230C

2. Fleischmann-Struzek C, Goldfarb DM, Schlattmann P, Schlapbach LJ, Reinhart K, Kissoon N: The global burden of paediatric and neonatal sepsis: a systematic review. Lancet Respir Med. 2018, 6:223-230. 10.1016/S2213-2600(18)30063-8

3. Ruth A, McCracken CE, Fortenberry JD, Hall M, Simon HK, Hebbar KB: Pediatric severe sepsis: current trends and outcomes from the Pediatric Health Information Systems database. Pediatr Crit Care Med. 2014, 15:828-838. 10.1097/PCC.0000000000000254

4. Schlapbach LJ, MacLaren G, Festa M, et al.: Prediction of pediatric sepsis mortality within $1 \mathrm{~h}$ of intensive care admission. Intensive Care Med. 2017, 43:1085-1096. 10.1007/s00134-017-4701-8

5. Weiss SL, Balamuth F, Hensley J, et al.: The epidemiology of hospital death following pediatric severe sepsis: when, why, and how children with sepsis die. Pediatr Crit Care Med. 2017, 18:823-830. 10.1097/PCC.0000000000001222

6. Morin L, Ray S, Wilson C, et al.: Refractory septic shock in children: a European Society of Paediatric and Neonatal Intensive Care definition. Intensive Care Med. 2016, 42:1948-1957. 10.1007/s00134-016-4574-2

7. Leclerc F, Leteurtre S, Duhamel A, et al.: Cumulative influence of organ dysfunctions and septic state on mortality of critically ill children. Am J Respir Crit Care Med. 2005, 171:348-353. 10.1164/rccm.200405$6300 \mathrm{C}$

8. Bai Z, Zhu X, Li M, et al.: Effectiveness of predicting in-hospital mortality in critically ill children by assessing blood lactate levels at admission. BMC Pediatr. 2014, 14:83-2014. 10.1186/1471-2431-14-83

9. Weiss SL, Peters MJ, Alhazzani W, et al.: Surviving sepsis campaign international guidelines for the management of septic shock and sepsis-associated organ dysfunction in children. Intensive Care Med. 2020, 46:10-67. 10.1007/s00134-019-05878-6

10. Singer M, Deutschman CS, Seymour CW, et al.: The third international consensus definitions for sepsis and septic shock (Sepsis-3). JAMA. 2016, 315:801-810. 10.1001/jama.2016.0287

11. Prout AJ, Talisa VB, Carcillo JA, et al.: Children with chronic disease bear the highest burden of pediatric sepsis. J Pediatr. 2018, 199:194-199. 10.1016/j.jpeds.2018.03.056

12. Fjalstad JW, Stensvold HJ, Bergseng H, Simonsen GS, Salvesen B, Rønnestad AE, Klingenberg C: Early-onset sepsis and antibiotic exposure in term infants: a nationwide population-based study in Norway. Pediatr Infect Dis J. 2016, 35:1-6.

13. Agyeman PKA, Schlapbach LJ, Giannoni E, et al.: Epidemiology of blood culture-proven bacterial sepsis in children in Switzerland: a population-based cohort study. Lancet Child Adolesc Health. 2017, 1:124-133. 10.1016/S2352-4642(17)30010-X

14. McMullan BJ, Bowen A, Blyth CC, et al.: Epidemiology and mortality of Staphylococcus aureus bacteremia in Australian and New Zealand children. JAMA Pediatr. 2016, 170:979-986. 10.1001/jamapediatrics.2016.1477

15. Straney L, Clements A, Parslow RC, et al.: Paediatric Index of Mortality 3: an updated model for predicting mortality in pediatric intensive care. Pediatr Crit Care Med. 2013, 14:673-681. 10.1097/PCC.0b013e31829760cf

16. Goldstein B, Giroir B, Randolph A: International pediatric sepsis consensus conference: definitions for sepsis and organ dysfunction in pediatrics. Pediatr Crit Care Med. 2005, 6:2-8. 10.1097/01.PCC.0000149131.72248.E6

17. Jolley RJ, Quan H, Jetté N, et al.: Validation and optimisation of an ICD-10-coded case definition for sepsis using administrative health data. BMJ Open. 2015, 5:e009487. 10.1136/bmjopen-2015-009487

18. Feudtner C, Feinstein JA, Zhong W, Hall M, Dai D: Pediatric complex chronic conditions classification system version 2: updated for ICD-10 and complex medical technology dependence and transplantation. BMC Pediatr. 2014, 14:199. 10.1186/1471-2431-14-199

19. Phua J, Ngerng W, See K, et al.: Characteristics and outcomes of culture-negative versus culture-positive severe sepsis. Crit Care. 2013, 17:R202. 10.1186/cc12896

20. Lin JC, Spinella PC, Fitzgerald JC, et al.: New or progressive multiple organ dysfunction syndrome in pediatric severe sepsis: a sepsis phenotype with higher morbidity and mortality. Pediatr Crit Care Med. 2017, 18:8-16. 10.1097/PCC.0000000000000978

21. Gupta S, Sakhuja A, Kumar G, McGrath E, Nanchal RS, Kashani KB: Culture-negative severe sepsis: nationwide trends and outcomes. Chest. 2016, 150:1251-1259. 10.1016/j.chest.2016.08.1460 